11. Getting your dissertation done is extremely important. You're very unlikely to get a good job without a Ph.D. in hand. However, getting articles accepted for publication is also very important. Most of my colleagues advise you to convert the appropriate sections of your dissertation into articles only after the dissertation is finished. I think you should do so simultaneously, as you complete them. That way, not only do you get possible published articles but you also get valuable professional feedback.

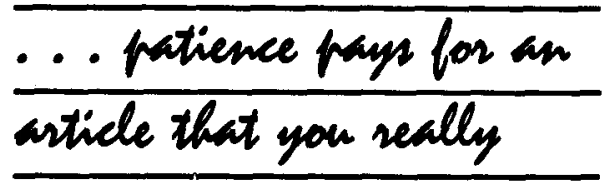

\section{believe in.}

12. The more people you show your work to, the more people there are who might read it. But all advice is not equal, and writing a thesis should not resemble a popularity/public opinion poll. Remember, it's your thesis.

13. Choose an advisor whom you respect both as an intellect and as a human being. (Not necessarily in that order.)

14. Before going on a job interview learn the names (and even read some of the articles) of the people who are going to interview you. There's nothing so embarrassing as being introduced to somebody with no idea who they are, when they think that everybody should know who they are and why they're "famous."

15. In going on a job interview, there is only one rule: Be yourself. When you are interviewing be warned, however, that many (if not most) of the faculty at the institution where you sent your vita and publications, didn't read them. Thus, reminding people of who you are and what you do can't hurt.

\section{Notes}

* I am indebted to jim Danziger for helpful comments, also the original idea for item $\mathrm{fl}$ | was his. Items $/ 12$ and $f 13$ were added by Kristen Monroe as was a portion of item / 15. However, the views expressed in this essay are solely the responsibility of the author.

\section{Congressional and Presidential Scholars: Some Basic Traits*}

\author{
Christopher J. Bosso \\ Northeastern University
}

\section{Introduction}

Accustomed as we students of American politics are to training our analytical tools on various segments of the national population, we rarely use them on ourselves. Perhaps we are not all that interested in knowing more about who we are, either as people or as scholars. Perhaps the academic reward system finds little worth in such studies. Perhaps we really do not want to know whether there exist any noticeable biases among those whose scholarship and teaching influence generations of students and politicians.

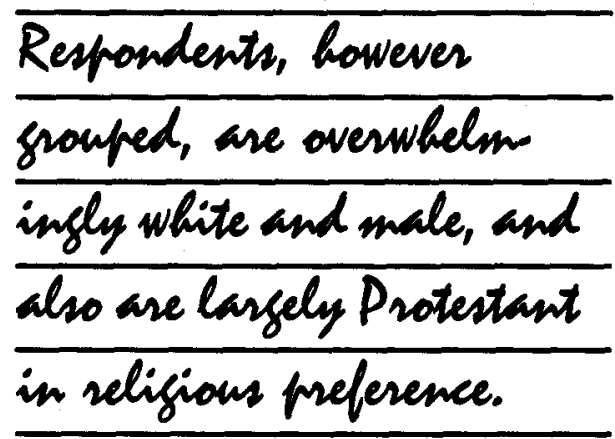

Whatever is the case, and it might well be a mixture of all three reasons, the purpose of this essay is to present summary data on some basic personal and professional traits of two scholarly cohorts within 
the field of American national politics: members of either the Legislative Studies Section or the Presidency Research Group of the American Political Science Association. The data shown here were taken from a mail survey sent to approximately 700 members of either group in August 1987. The primary purpose of the survey was to assess scholarly views about Congress in light of the social, political, and institutional changes of the past generation, but the opportunity also was taken to query respondents on their personal traits, current professional status, political attachments, and broad attitudes on a select array of issues. Those polled comprised the entire membership of each section as represented by APSA mailing lists, and, of those to whom surveys were sent, $51 \%(361$ ) completed and returned the questionnaire.

\section{Alout $80 \%$ are below the}

\section{age of fifty, and 66\% \\ cussently ase in their}

\section{thisties on forties.}

Any conclusions to be made about all congressional and presidential scholarsmuch less all American political scientistsmust be tempered, of course, by the caveat that membership in either the LSS or PRG, and the decision to complete this survey, was entirely voluntary. Furthermore, members of either section are likely to be more established scholars in residence at more research-oriented departments then might be true for all political scientists who teach about Congress and the presidency. Nonetheless, the data shown here do give some greater insight into the characteristics of those who, because of their active research efforts and greater participation in APSA activities, arguably lead the way in influencing how this discipline portrays legislative and executive politics to both our students and the world at large.

\section{Who We Are}

Respondents, however grouped, are overwheimingly white and male, and also are largely Protestant in religious preference. Whether the notably low percentage of female or nonwhite scholars shown here reflects the discipline as a whole is, of course, the compelling question. According to the data calculated from the APSA's 1986 Guide to Graduate Study in Political Science, for example, women accounted for approximately $28 \%$ of all students in doctoral programs at the time, while blacks represented about $6 \%$ of the total. Many of these no doubt were foreigners who accounted for approximately $26 \%$ of all doctoral students, and who are far less likely to be scholars of American politics anyway (pp. 395-98). Female and minority scholars as a whole also may be likelier to focus on the comparative or international relations fields. Whatever the case, female and minority representation among respondents is notably low, and the paucity of nonwhite respondents found here makes any further comparisons based on racial characteristics useless.

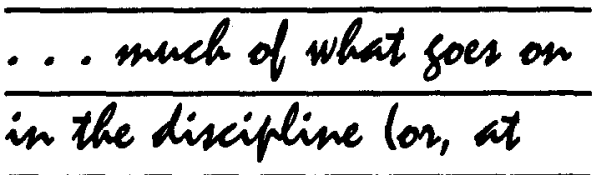
least, in these two $\frac{\text { onganized rections), does }}{\text { not travel much to part }}$ time faculty on those in the community colleges.

Respondents in both subsamples also are relatively young. About $80 \%$ are below the age of fifty, and $66 \%$ currently are in their thirties or forties. Female respondents as a whole are noticeably younger than their male counterparts, 


\section{Congressional and Presidential Scholars}

probably reflecting the relatively recent influx of women (apparently on a modest scale) into these two subfields (see below). The age disparity between male and female respondents is most obvious among presidency scholars, though, to be clear about it, there were too few females in the PRG subsample to make conclusive judgments.

\section{Professional Status}

Almost $80 \%$ of those responding have earned a doctorate, a percentage that is pretty constant across subsamples. There are, however, obvious differences between males and females. For example, female respondents were, in percentage

Table I. Gender, Race, and Religious Preference ( $\%$ and N)

\begin{tabular}{|c|c|c|c|c|c|c|}
\hline \multirow[b]{2}{*}{$\begin{array}{l}\text { Male } \\
\text { Female } \\
\text { Missing }\end{array}$} & \multicolumn{2}{|c|}{ All } & \multicolumn{2}{|c|}{ LSS } & \multicolumn{2}{|c|}{ PRG } \\
\hline & $\begin{array}{l}83.3 \% \\
16.7 \%\end{array}$ & $\begin{array}{r}299 \\
60 \\
2\end{array}$ & $\begin{array}{l}81.7 \% \\
18.3 \%\end{array}$ & $\begin{array}{r}196 \\
44 \\
1\end{array}$ & $\begin{array}{l}86.6 \% \\
13.4 \%\end{array}$ & $\begin{array}{r}103 \\
16 \\
1\end{array}$ \\
\hline Total & $100.0 \%$ & 361 & $100.0 \%$ & 241 & $100.0 \%$ & 120 \\
\hline $\begin{array}{l}\text { Race } \\
\text { Caucasian } \\
\text { Black } \\
\text { Hispanic } \\
\text { Native } \\
\text { Asian } \\
\text { Other } \\
\text { Missing }\end{array}$ & $\begin{array}{r}93.8 \% \\
0.8 \% \\
1.7 \% \\
1.4 \% \\
0.3 \% \\
2.0 \%\end{array}$ & $\begin{array}{r}331 \\
3 \\
6 \\
5 \\
1 \\
7 \\
8\end{array}$ & $\begin{array}{r}93.3 \% \\
0.4 \% \\
2.1 \% \\
1.3 \% \\
0.4 \% \\
2.5 \%\end{array}$ & $\begin{array}{r}222 \\
1 \\
5 \\
3 \\
1 \\
6 \\
3\end{array}$ & $\begin{array}{r}94.8 \% \\
1.7 \% \\
0.9 \% \\
1.7 \% \\
0.0 \% \\
0.9 \%\end{array}$ & $\begin{array}{r}109 \\
2 \\
1 \\
2 \\
0 \\
1 \\
5\end{array}$ \\
\hline Total & $100.0 \%$ & 361 & $100.0 \%$ & 241 & $100.0 \%$ & 120 \\
\hline $\begin{array}{l}\text { Moles } \\
\text { White } \\
\text { Black } \\
\text { Hispanic } \\
\text { Native } \\
\text { Asian } \\
\text { Other } \\
\text { Missing }\end{array}$ & $\begin{array}{r}94.2 \% \\
0.7 \% \\
1.7 \% \\
1.4 \% \\
0.3 \% \\
1.7 \%\end{array}$ & $\begin{array}{r}275 \\
2 \\
5 \\
4 \\
1 \\
5 \\
7\end{array}$ & $\begin{array}{r}93.8 \% \\
0.0 \% \\
2.1 \% \\
1.0 \% \\
0.5 \% \\
2.6 \%\end{array}$ & $\begin{array}{r}182 \\
0 \\
4 \\
2 \\
1 \\
5 \\
2\end{array}$ & $\begin{array}{r}94.9 \% \\
2.0 \% \\
1.0 \% \\
2.0 \% \\
0.0 \% \\
0.0 \%\end{array}$ & $\begin{array}{r}93 \\
2 \\
1 \\
2 \\
0 \\
0 \\
5\end{array}$ \\
\hline Total & $100.0 \%$ & 299 & $100.0 \%$ & 196 & $100.0 \%$ & 103 \\
\hline $\begin{array}{l}\text { Females } \\
\text { White } \\
\text { Black } \\
\text { Hispanic } \\
\text { Native } \\
\text { Asian } \\
\text { Other } \\
\text { Missing }\end{array}$ & $\begin{array}{r}91.7 \% \\
1.7 \% \\
1.7 \% \\
1.7 \% \\
0.0 \% \\
3.3 \%\end{array}$ & $\begin{array}{r}55 \\
1 \\
1 \\
1 \\
0 \\
2 \\
0\end{array}$ & $\begin{array}{r}90.9 \% \\
2.3 \% \\
2.3 \% \\
2.3 \% \\
0.0 \% \\
2.3 \%\end{array}$ & $\begin{array}{r}40 \\
1 \\
1 \\
1 \\
0 \\
1 \\
0\end{array}$ & $\begin{array}{r}93.8 \% \\
0.0 \% \\
0.0 \% \\
0.0 \% \\
0.0 \% \\
6.3 \%\end{array}$ & $\begin{array}{r}15 \\
0 \\
0 \\
0 \\
0 \\
1 \\
0\end{array}$ \\
\hline Total & $100.0 \%$ & 60 & $100.0 \%$ & 44 & $100.0 \%$ & 16 \\
\hline $\begin{array}{l}\text { Religious Pref } \\
\text { Protestant } \\
\text { Jewish } \\
\text { Catholic } \\
\text { Other } \\
\text { None } \\
\text { No Answer }\end{array}$ & $\begin{array}{r}47.3 \% \\
13.2 \% \\
14.6 \% \\
3.9 \% \\
20.8 \%\end{array}$ & $\begin{array}{r}168 \\
47 \\
52 \\
14 \\
74 \\
6\end{array}$ & $\begin{array}{r}47.1 \% \\
13.9 \% \\
15.1 \% \\
3.4 \% \\
20.6 \%\end{array}$ & $\begin{array}{r}112 \\
33 \\
36 \\
8 \\
49 \\
3\end{array}$ & $\begin{array}{r}47.9 \% \\
12.0 \% \\
13.7 \% \\
5.1 \% \\
21.4 \%\end{array}$ & $\begin{array}{r}56 \\
14 \\
16 \\
6 \\
25 \\
3\end{array}$ \\
\hline Total & $100.0 \%$ & 361 & $100.0 \%$ & 241 & $100.0 \%$ & 120 \\
\hline
\end{tabular}


Table 2. Age (\% and $N)$

\begin{tabular}{lrrrrrr}
\hline & \multicolumn{2}{c}{ All } & \multicolumn{2}{c}{ LSS } & \multicolumn{2}{c}{ PRG } \\
\hline 20 to 29 years & $13.9 \%$ & 49 & $15.3 \%$ & 36 & $11.1 \%$ & 13 \\
30 to 39 years & $32.0 \%$ & 113 & $30.9 \%$ & 73 & $34.2 \%$ & 40 \\
40 to 49 years & $34.0 \%$ & 120 & $37.7 \%$ & 89 & $26.5 \%$ & 31 \\
50 to 59 years & $15.6 \%$ & 55 & $13.1 \%$ & 31 & $20.5 \%$ & 24 \\
$60+$ years & $4.5 \%$ & 16 & $3.0 \%$ & 7 & $7.7 \%$ & 9 \\
Missing & & 8 & & 5 & & 3 \\
Total & $100.0 \%$ & 361 & $100.0 \%$ & 241 & $100.0 \%$ & 120 \\
Males & & & & & & \\
20 to 29 years & $11.9 \%$ & 35 & $12.4 \%$ & 24 & $10.9 \%$ & 11 \\
30 to 39 years & $30.5 \%$ & 90 & $30.4 \%$ & 59 & $30.7 \%$ & 31 \\
40 to 49 years & $34.6 \%$ & 102 & $38.1 \%$ & 74 & $27.7 \%$ & 28 \\
50 to 59 years & $18.3 \%$ & 54 & $15.5 \%$ & 30 & $23.8 \%$ & 24 \\
$60+$ years & $4.7 \%$ & 14 & $3.6 \%$ & 7 & $6.9 \%$ & 7 \\
Missing & & 4 & & 2 & & 2 \\
Total & $100.0 \%$ & 299 & $100.0 \%$ & 196 & $100.0 \%$ & 103 \\
Females & & & & & & \\
20 to 29 years & $24.1 \%$ & 14 & $28.6 \%$ & 12 & $12.5 \%$ & 2 \\
30 to 39 years & $39.7 \%$ & 23 & $33.3 \%$ & 14 & $56.3 \%$ & 9 \\
40 to 49 years & $31.0 \%$ & 18 & $35.7 \%$ & 15 & $18.8 \%$ & 3 \\
50 to 59 years & $1.7 \%$ & 1 & $2.4 \%$ & 1 & $0.0 \%$ & 0 \\
$60+$ years & $3.4 \%$ & 2 & $0.0 \%$ & 0 & $12.5 \%$ & 2 \\
Missing & & 2 & & 2 & & 0 \\
Total & $100.0 \%$ & 60 & $100.0 \%$ & 44 & $100.0 \%$ & 16 \\
\hline
\end{tabular}

Table 3. Current Educational Status

\begin{tabular}{lrrrrrr}
\hline & All & & LSS & \multicolumn{2}{c}{ PRG } \\
\hline Degree Attained & & & & & & \\
Bachelors & $5.9 \%$ & 21 & $7.1 \%$ & 17 & $3.4 \%$ & 4 \\
Masters & $13.0 \%$ & 46 & $13.0 \%$ & 31 & $12.9 \%$ & 15 \\
Doctorate & $78.8 \%$ & 279 & $77.7 \%$ & 185 & $81.0 \%$ & 94 \\
Other & $2.3 \%$ & 8 & $2.1 \%$ & 5 & $2.6 \%$ & 3 \\
Missing & & 7 & & 3 & & 4 \\
$\quad$ Total & $100.0 \%$ & 361 & $100.0 \%$ & 241 & $100.0 \%$ & 120 \\
Males & & & & & & \\
Bachelors & $5.5 \%$ & 16 & $6.2 \%$ & 12 & $4.0 \%$ & 4 \\
Masters & $11.3 \%$ & 33 & $11.3 \%$ & 22 & $11.1 \%$ & 11 \\
Doctorate & $80.9 \%$ & 237 & $79.9 \%$ & 155 & $82.8 \%$ & 82 \\
Other & $2.4 \%$ & 7 & $2.6 \%$ & 5 & $2.0 \%$ & 2 \\
Missing & & 6 & & 2 & & 4 \\
$\quad$ Total & $100.0 \%$ & 299 & $100.0 \%$ & 196 & $100.0 \%$ & 103 \\
Females & & & & & & \\
Bachelors & $8.3 \%$ & 5 & $11.4 \%$ & 5 & $0.0 \%$ & 0 \\
Masters & $21.7 \%$ & 13 & $20.5 \%$ & 9 & $25.0 \%$ & 4 \\
Doctorate & $1.3 \%$ & 41 & $68.2 \%$ & 30 & $68.8 \%$ & 11 \\
Other & $1.7 \%$ & 1 & $0.0 \%$ & 0 & $6.3 \%$ & 1 \\
Missing & & 0 & & 0 & & 0 \\
$\quad$ Total & $100.0 \%$ & 60 & $100.0 \%$ & 44 & $100.0 \%$ & 16 \\
\hline
\end{tabular}


terms, more likely to have attained only as far as the M.A. degree, but, more than anything else, this probably is the result of their relative youth and current stage in the academic career path.

\section{Respondents largely are \\ more liberal and aligned}

more with the Democratic

\section{party, tend to cast their \\ votes along straight party \\ lines, and voted over-}

whelmingly in 1984 for

\section{Walter Mondale.}

Most ( $86 \%)$ who earned the doctorate did so since 1965, with the peak reached during the 1970s. Female respondents accounted for only $10 \%$ of all who completed the Ph.D. prior to 1980. Since then, however, women have accounted for $30 \%$ of all doctorates earned, and almost $60 \%$ of all female respondents who completed the doctorate did so this decade, particularly during the past five years. This trend probably accounts for the far more junior academic status of women relative to their male counterparts (see below).

As Table 4 shows, the greatest number of doctorates were earned at the University of Wisconsin at Madison (15), followed closely by Yale (14), Michigan (II), and Cornell (II). For women, however, Minnesota, Florida State, and Johns Hopkins led the way (modestly). The latter two schools, which produced totals of seven and five doctorates respectively, do not appear among the top ten of all institutions mentioned.

The overwhelming majority of all respondents ( $87 \%$ ) currently are affiliated with institutions of higher learning, while those not so linked typically cited service in government at some level as an occupation. Among those currently affiliated with
Table 4. Top Ten Sources of Doctorates, 1950-1988

\begin{tabular}{lcc}
\hline & Males & Females \\
\hline Wisconsin at Madison & 14 & 1 \\
Yale & 14 & 0 \\
Michigan & 9 & 2 \\
Cornell & 9 & 2 \\
Chicago & 10 & 0 \\
Harvard & 9 & 0 \\
Michigan State & 8 & 0 \\
lowa & 7 & 1 \\
Minnesota & 5 & 3 \\
Syracuse & 7 & 1 \\
\hline
\end{tabular}

universities or colleges (not including graduate students), a majority reside in state schools, with private, non-sectarian institutions the second most common designation. This is constant across subsamples, but note also the differences between male and female respondents: women are more likely to be affiliated with private universities or colleges (either sectarian or non-sectarian) than with state schools.

Respondents of either gender-93\% for males, $84 \%$ for females-are full-time faculty, and $96 \%$ of all respondents teach in four-year institutions. All of this leaves one to suspect that much of what goes on in the discipline (or, at least, in these two organized sections), does not travel much to part-time faculty or those in the community colleges.

More than half of all respondents teach in departments that offer the doctorate as the highest degree, but there seems to be a noticeable distinction between congressional and presidential scholars in this regard: more than half of respondents in the PRG subsample teach in nondoctoral programs. Female respondents, in percentage terms, are more likely than males to be part of doctoral programs, but whether this reflects strong affirmative action efforts by these departments or simply is an artifact of this sample is unknown.

As a group, respondents overwhelmingly are a more senior and tenured lot. Only 20\% are in the assistant professor ranks, half of those who currently are full professors but almost equal in percentage terms to those in the associate ranks. 
Table 5. Type of School

\begin{tabular}{|c|c|c|c|c|c|c|}
\hline \multirow[b]{2}{*}{$\begin{array}{l}\text { State } \\
\text { Private-Sectarian } \\
\text { Private-Non-Sectarian } \\
\text { Municipal } \\
\text { Military } \\
\text { Other } \\
\text { Missing }\end{array}$} & \multicolumn{2}{|c|}{ All } & \multicolumn{2}{|c|}{ LSS } & \multicolumn{2}{|c|}{ PRG } \\
\hline & $\begin{array}{l}63.4 \% \\
11.0 \% \\
23.3 \% \\
1.0 \% \\
1.3 \% \\
0.0 \%\end{array}$ & $\begin{array}{r}196 \\
34 \\
72 \\
3 \\
4 \\
0 \\
52\end{array}$ & $\begin{array}{r}63.9 \% \\
8.9 \% \\
24.8 \% \\
1.5 \% \\
1.0 \% \\
0.0 \%\end{array}$ & $\begin{array}{r}129 \\
18 \\
50 \\
3 \\
2 \\
0 \\
39\end{array}$ & $\begin{array}{r}62.6 \% \\
15.0 \% \\
20.6 \% \\
0.0 \% \\
1.9 \% \\
0.0 \%\end{array}$ & $\begin{array}{r}67 \\
16 \\
22 \\
0 \\
2 \\
0 \\
13\end{array}$ \\
\hline Total & $100.0 \%$ & 361 & $100.0 \%$ & 241 & $100.0 \%$ & 120 \\
\hline $\begin{array}{l}\text { Males } \\
\text { State } \\
\text { Private-Sectarian } \\
\text { Private-Non-Sectarian } \\
\text { Municipal } \\
\text { Military } \\
\text { Other } \\
\text { Missing }\end{array}$ & $\begin{array}{r}66.5 \% \\
9.6 \% \\
21.5 \% \\
1.2 \% \\
1.2 \% \\
0.0 \%\end{array}$ & $\begin{array}{r}173 \\
25 \\
56 \\
3 \\
3 \\
0 \\
39\end{array}$ & $\begin{array}{r}66.1 \% \\
8.3 \% \\
22.6 \% \\
1.8 \% \\
1.2 \% \\
0.0 \%\end{array}$ & $\begin{array}{r}111 \\
14 \\
38 \\
3 \\
2 \\
0 \\
28\end{array}$ & $\begin{array}{l}67.4 \% \\
12.0 \% \\
19.6 \% \\
1.1 \% \\
0.0 \% \\
0.0 \%\end{array}$ & $\begin{array}{r}62 \\
11 \\
18 \\
1 \\
0 \\
0 \\
11\end{array}$ \\
\hline Total & $100.0 \%$ & 299 & $100.0 \%$ & 196 & $100.0 \%$ & 103 \\
\hline $\begin{array}{l}\text { Females } \\
\text { State } \\
\text { Private-Sectarian } \\
\text { Private-Non-Sectarian } \\
\text { Municipal } \\
\text { Military } \\
\text { Other } \\
\text { Missing }\end{array}$ & $\begin{array}{r}47.9 \% \\
18.8 \% \\
31.3 \% \\
0.0 \% \\
2.1 \% \\
0.0 \%\end{array}$ & $\begin{array}{r}23 \\
9 \\
15 \\
0 \\
1 \\
0 \\
12\end{array}$ & $\begin{array}{r}52.9 \% \\
11.8 \% \\
35.3 \% \\
0.0 \% \\
0.0 \% \\
0.0 \%\end{array}$ & $\begin{array}{r}18 \\
4 \\
12 \\
0 \\
0 \\
0 \\
10\end{array}$ & $\begin{array}{l}35.7 \% \\
35.7 \% \\
21.4 \% \\
7.1 \% \\
0.0 \% \\
0.0 \%\end{array}$ & $\begin{array}{l}5 \\
5 \\
3 \\
1 \\
0 \\
0 \\
2\end{array}$ \\
\hline Total & $100.0 \%$ & 60 & $100.0 \%$ & 44 & $100.0 \%$ & 16 \\
\hline
\end{tabular}

Table 6. Highest Degree Offered by Respondent's Department

\begin{tabular}{lrrrrrr}
\hline & \multicolumn{2}{c}{ All } & \multicolumn{2}{c}{ LSS } & \multicolumn{2}{c}{ PRG } \\
\hline Bachelors & $24.8 \%$ & 77 & $19.3 \%$ & 39 & $35.2 \%$ & 38 \\
Masters & $19.0 \%$ & 59 & $19.3 \%$ & 39 & $18.5 \%$ & 20 \\
Doctorate & $54.5 \%$ & 169 & $58.9 \%$ & 119 & $46.3 \%$ & 50 \\
Other & $1.6 \%$ & 5 & $2.5 \%$ & 5 & $0.0 \%$ & 0 \\
NA & $100.0 \%$ & 361 & $100.0 \%$ & 241 & $100.0 \%$ & 120 \\
$\quad$ Total & & & & & & \\
Males & $23.6 \%$ & 64 & $19.6 \%$ & 33 & $30.1 \%$ & 31 \\
Bachelors & $19.6 \%$ & 53 & $20.2 \%$ & 34 & $18.4 \%$ & 19 \\
Masters & $51.3 \%$ & 139 & $57.1 \%$ & 96 & $41.7 \%$ & 43 \\
Doctorate & $5.5 \%$ & 15 & $3.0 \%$ & 5 & $9.7 \%$ & 10 \\
Other & & 28 & & 28 & & 0 \\
NA & $100.0 \%$ & 299 & $100.0 \%$ & 196 & $100.0 \%$ & 103 \\
$\quad$ Total & & & & & & \\
Females & $27.1 \%$ & 13 & $17.6 \%$ & 6 & $50.0 \%$ & 7 \\
Bachelors & $10.4 \%$ & 5 & $14.7 \%$ & 5 & $0.0 \%$ & 0 \\
Masters & $62.5 \%$ & 30 & $67.6 \%$ & 23 & $50.0 \%$ & 7 \\
Doctorate & $0.0 \%$ & 0 & $0.0 \%$ & 0 & $0.0 \%$ & 0 \\
Other & & 12 & & 10 & & 2 \\
NA & $100.0 \%$ & 60 & $100.0 \%$ & 44 & $100.0 \%$ & 16 \\
$\quad$ Total & & & & & & \\
\hline
\end{tabular}


Congressional and Presidential Scholars

Table 7. Current Rank

\begin{tabular}{|c|c|c|c|c|c|c|}
\hline \multirow[b]{2}{*}{$\begin{array}{l}\text { Instructor/Lecturer } \\
\text { Assistant Professor } \\
\text { Associate Professor } \\
\text { Professor } \\
\text { Graduate Student } \\
\text { Fellow } \\
\text { Administrator } \\
\text { Missing }\end{array}$} & \multicolumn{2}{|c|}{ All } & \multicolumn{2}{|c|}{ LSS } & \multicolumn{2}{|c|}{ PRG } \\
\hline & $\begin{array}{r}4.9 \% \\
20.1 \% \\
21.7 \% \\
40.1 \% \\
10.5 \% \\
1.0 \% \\
1.6 \%\end{array}$ & $\begin{array}{r}15 \\
61 \\
66 \\
122 \\
32 \\
3 \\
5 \\
57\end{array}$ & $\begin{array}{r}3.1 \% \\
20.4 \% \\
23.0 \% \\
39.3 \% \\
11.2 \% \\
0.5 \% \\
2.6 \%\end{array}$ & $\begin{array}{r}6 \\
40 \\
45 \\
77 \\
22 \\
1 \\
5 \\
45\end{array}$ & $\begin{array}{r}8.3 \% \\
19.4 \% \\
19.4 \% \\
41.7 \% \\
9.3 \% \\
1.9 \% \\
0.0 \%\end{array}$ & $\begin{array}{r}9 \\
21 \\
21 \\
45 \\
10 \\
2 \\
0 \\
12\end{array}$ \\
\hline Total & $100.0 \%$ & 361 & $100.0 \%$ & 241 & $100.0 \%$ & 120 \\
\hline $\begin{array}{l}\text { Males } \\
\text { Instructor/Lecturer } \\
\text { Assistant Professor } \\
\text { Associate Professor } \\
\text { Professor } \\
\text { Graduate Student } \\
\text { Fellow } \\
\text { Administrator } \\
\text { Missing/NA }\end{array}$ & $\begin{array}{r}3.5 \% \\
17.1 \% \\
23.3 \% \\
44.7 \% \\
8.6 \% \\
1.2 \% \\
1.6 \%\end{array}$ & $\begin{array}{r}9 \\
44 \\
60 \\
115 \\
22 \\
3 \\
4 \\
42\end{array}$ & $\begin{array}{r}2.4 \% \\
17.7 \% \\
24.4 \% \\
43.9 \% \\
8.5 \% \\
0.6 \% \\
2.4 \%\end{array}$ & $\begin{array}{r}4 \\
29 \\
40 \\
72 \\
14 \\
1 \\
4 \\
32\end{array}$ & $\begin{array}{r}5.4 \% \\
16.1 \% \\
21.5 \% \\
46.2 \% \\
8.6 \% \\
2.2 \% \\
0.0 \%\end{array}$ & $\begin{array}{r}5 \\
15 \\
20 \\
43 \\
8 \\
2 \\
0 \\
10\end{array}$ \\
\hline Total & $100.0 \%$ & 299 & $100.0 \%$ & 196 & $100.0 \%$ & 103 \\
\hline $\begin{array}{l}\text { Femoles } \\
\text { linstructor/Lecturer } \\
\text { Assistant Professor } \\
\text { Associate Professor } \\
\text { Professor } \\
\text { Graduate Student } \\
\text { Fellow } \\
\text { Administrator } \\
\text { Missing/NA }\end{array}$ & $\begin{array}{r}13.0 \% \\
37.0 \% \\
13.0 \% \\
13.0 \% \\
21.7 \% \\
0.0 \% \\
2.2 \%\end{array}$ & $\begin{array}{r}6 \\
17 \\
6 \\
6 \\
10 \\
0 \\
1 \\
14\end{array}$ & $\begin{array}{r}6.3 \% \\
34.4 \% \\
15.6 \% \\
15.6 \% \\
25.0 \% \\
0.0 \% \\
3.1 \%\end{array}$ & $\begin{array}{r}2 \\
11 \\
5 \\
5 \\
8 \\
0 \\
1 \\
12\end{array}$ & $\begin{array}{r}28.6 \% \\
42.9 \% \\
7.1 \% \\
7.1 \% \\
14.3 \% \\
0.0 \% \\
0.0 \%\end{array}$ & $\begin{array}{l}4 \\
6 \\
1 \\
1 \\
2 \\
0 \\
0 \\
2\end{array}$ \\
\hline Total & $100.0 \%$ & 60 & $100.0 \%$ & 44 & $100.0 \%$ & 16 \\
\hline
\end{tabular}

Table 8. Current Tenure Status

\begin{tabular}{lrrrrrr}
\hline & \multicolumn{2}{c}{ All } & \multicolumn{2}{c}{ LSS } & \multicolumn{2}{c}{ PRG } \\
\hline Non-Tenure Track & $11.1 \%$ & 30 & $11.0 \%$ & 19 & $11.1 \%$ & $1 !$ \\
Tenure Track & $20.3 \%$ & 55 & $20.9 \%$ & 36 & $19.2 \%$ & 19 \\
Tenured & $68.6 \%$ & 186 & $68.0 \%$ & 117 & $69.7 \%$ & 69 \\
NA/Missing & & 90 & & 69 & & 21 \\
$\quad$ Total & $100.0 \%$ & 361 & $100.0 \%$ & 241 & $100.0 \%$ & 120 \\
Moles & & & & & & \\
Non-Tenure Track & $9.4 \%$ & 22 & $10.7 \%$ & 16 & $7.0 \%$ & 6 \\
Tenure Track & $17.0 \%$ & 40 & $17.4 \%$ & 26 & $16.3 \%$ & 14 \\
Tenuured & $73.6 \%$ & 173 & $71.8 \%$ & 107 & $76.7 \%$ & 66 \\
NA/Missing & & 64 & & 47 & & 17 \\
$\quad$ Total & $100.0 \%$ & 299 & $100.0 \%$ & 196 & $100.0 \%$ & 103 \\
Femoles & & & & & & \\
Non-Tenure Track & $22.9 \%$ & 8 & $13.0 \%$ & 3 & $41.7 \%$ & 5 \\
Tenure Track & $42.9 \%$ & 15 & $43.5 \%$ & 10 & $41.7 \%$ & 5 \\
Tenured & $34.3 \%$ & 12 & $43.5 \%$ & 10 & $16.7 \%$ & 2 \\
NA/Missing & & 25 & & 21 & & 4 \\
$\quad$ Total & $100.0 \%$ & 60 & $100.0 \%$ & 44 & $100.0 \%$ & 16 \\
\hline & & & & & &
\end{tabular}


There is a clear difference between males and females in this regard, with women at present more likely to populate the assistant professor and instructor/lecturer ranks. This is reflected again in Table 8 , where women currently are both far less tenured and likelier to hold non-tenure track positions. The differences are most acute for women members of the PRG, but, again, the small subsample size offers no definitive statements.

The relative seniority of respondents in either subsample is reflected in approximate university salaries, which are skewed heavily toward the higher end of the scale. Male respondents are more highly paid

Table 9. Current University Salary

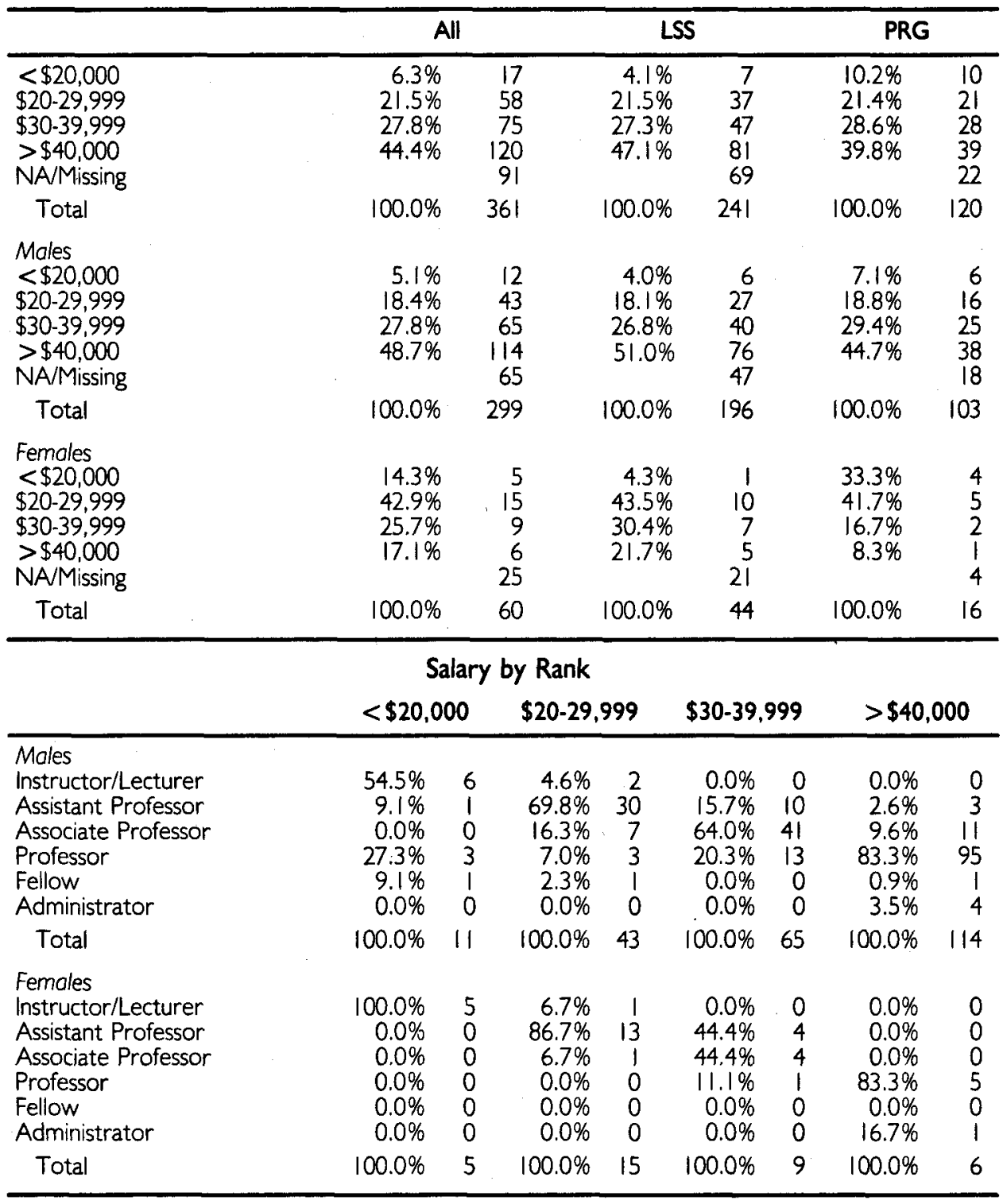


than their female colleagues, but this disparity apparently is not due to any systematic gender bias. Relative rank and tenure determines salary levels, for, as Table 9 shows, $83 \%$ of those of either gender earning more than $\$ 40,000$ held a tenured professor rank. Overall, for both males and females, the correlation between rank and salary is stronger than any other, even tenure. The possible exception to be seen here lies in the associate ranks, but the numbers here are too small to make any conclusive statements about gender bias in remuneration.

\section{Partisanship, Ideology and Voting Habits}

Respondents also were asked about their self-professed ideological leanings, partisan attachments, and voting records. Respondents largely are more liberal and aligned more with the Democratic party, tend to cast their votes along straight par. ty lines, and voted overwhelmingly in 1984 for Walter Mondale. There is no great difference in ideological leanings between the subsamples, though one can discern a slightly more liberal and Democratic cast among those who are members of the Legislative Studies Section.

Finally, to add a bit of whimsy to the whole thing, those surveyed in August 1987 were asked, in an open-ended question, to predict the 1988 Democratic and Republican nominees for president, as well as which candidate was likeliest to win the White House that year. Given the

Table 10. Ideology, Partisanship, and Vote Preferences

\begin{tabular}{|c|c|c|c|c|c|c|}
\hline \multirow[b]{2}{*}{$\begin{array}{l}\text { Ideological Preference } \\
\text { Very Conservative } \\
\text { Somewhat Conservative } \\
\text { Middle } \\
\text { Somewhat Liberal } \\
\text { Very Liberal } \\
\text { Other } \\
\text { NA }\end{array}$} & \multicolumn{2}{|c|}{. All } & \multicolumn{2}{|c|}{ LSS } & \multicolumn{2}{|c|}{ PRG } \\
\hline & $\begin{array}{r}0.6 \% \\
14.2 \% \\
17.8 \% \\
46.5 \% \\
17.3 \% \\
3.7 \%\end{array}$ & $\begin{array}{r}2 \\
50 \\
63 \\
164 \\
61 \\
13 \\
8\end{array}$ & $\begin{array}{r}0.8 \% \\
12.3 \% \\
16.1 \% \\
46.6 \% \\
19.9 \% \\
4.2 \%\end{array}$ & $\begin{array}{r}2 \\
29 \\
38 \\
110 \\
47 \\
10 \\
5\end{array}$ & $\begin{array}{c}0.0 \% \\
17.9 \% \\
21.4 \% \\
46.2 \% \\
12.0 \% \\
2.6 \%\end{array}$ & $\begin{array}{r}0 \\
21 \\
25 \\
54 \\
14 \\
3 \\
3\end{array}$ \\
\hline Total & $100.0 \%$ & 361 & $100.0 \%$ & 241 & $100.0 \%$ & 120 \\
\hline $\begin{array}{l}\text { Partisanship } \\
\text { Republican } \\
\text { Democrat } \\
\text { Independent } \\
\text { Other } \\
\text { NA }\end{array}$ & $\begin{array}{r}16.1 \% \\
69.5 \% \\
13.8 \% \\
0.6 \%\end{array}$ & $\begin{array}{r}57 \\
246 \\
49 \\
2 \\
7\end{array}$ & $\begin{array}{r}14.4 \% \\
72.5 \% \\
12.7 \% \\
0.4 \%\end{array}$ & $\begin{array}{r}34 \\
171 \\
30 \\
1 \\
5\end{array}$ & $\begin{array}{r}19.5 \% \\
63.6 \% \\
16.1 \% \\
0.8 \%\end{array}$ & $\begin{array}{r}23 \\
75 \\
19 \\
1 \\
2\end{array}$ \\
\hline Total & $100.0 \%$ & 361 & $100.0 \%$ & 241 & $100.0 \%$ & 120 \\
\hline $\begin{array}{l}\text { Voting Tendencies } \\
\text { Straight Republican } \\
\text { Straight Democrat } \\
\text { Split Ticket } \\
\text { No Vote } \\
\text { NA }\end{array}$ & $\begin{array}{r}8.2 \% \\
52.3 \% \\
36.6 \% \\
2.8 \%\end{array}$ & $\begin{array}{r}29 \\
184 \\
129 \\
10 \\
9\end{array}$ & $\begin{array}{r}7.3 \% \\
55.1 \% \\
35.0 \% \\
2.6 \%\end{array}$ & $\begin{array}{r}17 \\
129 \\
82 \\
6 \\
7\end{array}$ & $\begin{array}{r}10.2 \% \\
46.6 \% \\
39.8 \% \\
3.4 \%\end{array}$ & $\begin{array}{r}12 \\
55 \\
47 \\
4 \\
2\end{array}$ \\
\hline Total & $100.0 \%$ & 361 & $100.0 \%$ & 241 & $100.0 \%$ & 120 \\
\hline $\begin{array}{l}1984 \text { Vote } \\
\text { Reagan } \\
\text { Mondale } \\
\text { Other } \\
\text { No Vote } \\
\text { NA }\end{array}$ & $\begin{array}{r}19.8 \% \\
72.5 \% \\
1.7 \% \\
5.9 \%\end{array}$ & $\begin{array}{r}70 \\
256 \\
6 \\
21 \\
8\end{array}$ & $\begin{array}{r}17.8 \% \\
75.0 \% \\
0.4 \% \\
6.8 \%\end{array}$ & $\begin{array}{r}42 \\
177 \\
1 \\
16 \\
5\end{array}$ & $\begin{array}{r}23.9 \% \\
67.5 \% \\
4.3 \% \\
4.3 \%\end{array}$ & $\begin{array}{r}28 \\
79 \\
5 \\
5 \\
3\end{array}$ \\
\hline Total & $100.0 \%$ & 361 & $100.0 \%$ & 241 & $100.0 \%$ & 120 \\
\hline
\end{tabular}


sample's overwhelming support for President Mondale, any predictions made by those brave enough to venture a guess were taken with the appropriate grains of salt.

On reflection, perhaps these results should have been released much earlier, if only to save the nation a lot of time and money. Respondents selected George Bush and Michael Dukakis as their respective party nominees. While Bush was mentioned most frequently for the Republican nomination (for a $49 \%$ plurality), members of the Legislative Studies Section actually sided with Robert Dole over the former vice president by a slim margin. Dukakis, meanwhile, won a plurality of votes (32\%) among nine Democrats mentioned, followed not all that closely by Mario Cuomo, apparently despite his protestations to noncandidacy.

When all was said and done, respondents tagged George Bush as the eventual winner, with Dole mentioned next most frequently as the probable 4 lst president. Dukakis came in a distant third. So much for all that talk about a repeat of the election of 1960.

\section{About the Author}

Christopher ]. Bosso is assistant professor of political science at Northeastern University. $\mathrm{He}$ is author of Pesticides and Politics: The Life Cycle of a Public issue (1987).

\section{Note}

"Funds for this survey were provided by the Everett McKinley Dirksen Congressional Leadership Research Center and the Northeastern University Research and Scholarship Development Fund.

\section{Studying Latino Politics: The Development of the Latino National Political Survey}

\author{
F. Chris Garcia \\ University of New Mexico \\ John A. Garcia \\ University of Arizona
}

\section{Angelo Falcon}

Institute for Puerto Rican Policy, Inc. and

\section{Rodolfo O. de la Garza \\ University of Texas, Austin}

As the 1980s, the proclaimed "decade of the Hispanic," draw to a close, marking roughly three decades of research on Latino politics, political scientists are making major strides in refining methodologies appropriate to the exploration of Latino political values and behavior. A major step has been the development of the Latino National Political Survey (LNPS), the first national opinion survey research project focusing on Latino politics in the continental United States. In addition to providing basic baseline information on the content of Latino political values, attitudes, and behavior, major advances also will have been made through the development of the LNPS in improving the methodology of scientifically surveying this increasingly important but underresearched population group.

\section{Background}

In the spring of 1984, a group of four political scientists, the authors of this article, began to explore the possibility of conducting the first truly national political opinion study of Latinos. Initially they envisioned collaborating with one of the major national polling organizations to piggyback a Latino sample onto some of the organization's on-going national surveys. However, as this possibility was examined 